\title{
Two cases of inflammatory muscle disease presenting with raised serum concentrations of troponin $T$
}

\author{
J S Hamilton, P C Sharpe
}

J Clin Pathol 2005;58:1323-1324. doi: 10.1136/icp.2005.025734

Troponins T and I are highly sensitive markers of myocardial injury. However, non-cardiac disorders, such as pulmonary embolism, renal failure, subarachnoid haemorrhage, sepsis, eclampsia, chemotherapy, and inflammatory muscle conditions (dermatomyositis and polymyositis), can also result in raised serum troponin concentrations. This article describes two cases that occurred within a month of each other in Craigavon Area Hospital, whereby conditions unrelated to myocardial ischaemia resulted in raised concentrations of cardiac markers. The first patient, in retrospect, underwent unnecessary investigation as an inpatient in the cardiac ward. Experience gained from this case led to more appropriate consultation and management of the second patient.

$\mathrm{T}$ roponin $\mathrm{T}$ is a sensitive marker of ischaemia induced myocardial damage. However, despite its excellent specificity, there are increasing numbers of both nonischaemic cardiac and non-cardiac conditions associated with raised concentrations. ${ }^{1}$ We present two case reports of patients with inflammatory muscle disease where the possible diagnosis of acute myocardial infarction arose. These patients were treated in very different ways. In our review of the literature pertaining to the causes of raised troponin concentrations, there was little reference to inflammatory muscle disease as a potential cause. Indeed, most lists of causes of raised troponin concentrations do not include this possibility. ${ }^{1}$

"Despite its excellent specificity, there are increasing numbers of both non-ischaemic cardiac and non-cardiac conditions associated with raised troponin $T$ concentrations"

\section{CASE REPORTS}

\section{Patient 1}

Patient 1 was a 58 year old woman with type 2 diabetes mellitus (treated with insulin), polymyositis, and hypertension. Medications were as follows: Humalog Mix25, 42 units a.m., 4 units p.m.; mycophenolate mofetil (Cellcept), $500 \mathrm{mg}$ twice daily; prednisolone, $5 \mathrm{mg}$ daily; sodium alendronate, 70 mg weekly; lisinopril, $5 \mathrm{mg}$ daily; folic acid, $0.4 \mathrm{mg}$ daily; and Adcal-D3. She had good glycaemic control (glycated haemoglobin, $7.0 \%$ ) and no history of cardiovascular disease.

Her family doctor requested a creatine kinase (CK) activity measurement because she had been complaining of increased muscular aches and weakness. She had experienced no chest discomfort or other symptoms suggestive of coronary heart disease. Total CK was raised at 615 U/litre (Roche Diagnostics, Mannheim, Germany; reference interval,
1-195; intra-assay coefficient of variation (CV), 0.6\%). CK$\mathrm{MB}$ (MB isoenzyme) was also raised at $53 \mathrm{U}$ /litre (Roche Diagnostics immunoinhibition assay; reference interval, 1.025.0; intra-assay CV, 3.8\%), with a \%CK-MB/total CK of $8.6 \%$. According to the laboratory protocol, a request for serum troponin $\mathrm{T}$ concentration (Roche Diagnostics; intra-assay CV, $2.3 \%$ at $0.2 \mu \mathrm{g} /$ litre) was automatically generated and was found to be raised at $0.174 \mu \mathrm{g} / \mathrm{litre}$ (troponin $\mathrm{T}>0.1 \mu \mathrm{g} / \mathrm{litre}$ is consistent with myocardial damage).

The biomedical scientist telephoned these results to the patient's family doctor as is standard protocol. The patient was admitted to the cardiac ward for further investigations. Subsequently, her electrocardiogram was normal and an exercise stress test showed no evidence of myocardial ischaemia; echocardiography was also normal. The troponin $\mathrm{T}$ concentration remained raised 48 hours later, at $0.158 \mu \mathrm{g} /$ litre. The results were discussed with the consultant chemical pathologist, who noted several reports in the literature describing raised concentrations of cardiac markers, including troponin $\mathrm{T}$, in patients with inflammatory muscle disease. $^{2-4}$ This patient's raised CK-MB and troponin T were therefore explained on the basis of her active polymyositis.

\section{Patient 2 (four weeks later)}

Patient 2 was a 38 year old woman with dermatomyositis attending the dermatology outpatient department; she was treated with both prednisolone and azathioprine. She complained of a two week history of increased muscular symptoms; total CK was 3642 U/litre, CK-MB was $937 \mathrm{U} /$ litre, and \%CK-MB/total CK was $25.7 \%$. As in the previous case, the troponin $\mathrm{T}$ concentration was measured according to laboratory protocol and was raised at $3.12 \mu \mathrm{g} /$ litre. In this case, the consultant chemical pathologist was notified of the results by the laboratory staff and the case was discussed with the consultant dermatologist; he felt that the patient was unlikely to have ischaemic heart disease or to have suffered a myocardial infarction. She had experienced no chest pains and an electrocardiogram was normal. The increased troponin $\mathrm{T}$ and $\mathrm{CK}-\mathrm{MB}$ were believed to be secondary to active dermatomyositis. Two weeks later, the patient's total CK was 1840 U/litre, CK-MB was 458 U/litre, $\%$ CK-MB/total CK was $24.9 \%$, and the troponin $\mathrm{T}$ concentration remained raised at $2.9 \mu \mathrm{g} / \mathrm{litre}$. An echocardiogram performed several weeks later revealed mild left ventricular hypertrophy, mild dilatation of the left ventricle, and a trace of mitral regurgitation. The ejection fraction was normal at $65 \%$.

\section{DISCUSSION}

The measurement of troponins ( $\mathrm{T}$ and $\mathrm{I}$ ) has largely superseded CK/CK-MB analysis, and is thought to provide more sensitive and specific data for use in the diagnosis and

Abbreviations: $C K$, creatine kinase; $C V$, coefficient of variation; $M B$, $M B$ isoenzyme 


\section{Take home messages}

- We describe two patients with raised concentrations of cardiac markers (creatine kinase MB isoenzyme and troponin T) in the absence of acute myocardial damage, caused by inflammatory muscular disease

- The exact source of the raised cardiac markers is unclear and could indicate myocardial involvement by the inflammatory process or a non-cardiac (probable skeletal muscle) source

- Thus, it is important to consider inflammatory muscle disease as a cause of increased troponin $T$ in the absence of clinical evidence of an acute coronary ischaemic event

prognosis of acute coronary syndromes. ${ }^{56}$ However, it is important to note that myocardial ischaemia is not the only cause of raised troponin concentrations, and that there are many other cardiac and non-cardiac causes. ${ }^{1}$ Increased troponin $\mathrm{T}$, and to a lesser extent troponin I, concentrations have been reported previously in patients with inflammatory muscle disease. ${ }^{2-4}$ Indeed, raised concentrations of troponin T have been reported in other disorders of skeletal muscle, such as Duchenne muscular dystrophy. ${ }^{7}$ Therefore, troponin concentrations must be interpreted in their clinical context. ${ }^{8}$

In these two case reports, the source of the raised biochemical cardiac markers (CK-MB and troponin $\mathrm{T}$ ) is uncertain. However, there are several possible explanations. First, myocardial and pericardial involvement (cardiomyopathy, myocarditis, and pericarditis) is a recognised, although relatively rare, feature in patients with dermatomyositis and polymyositis. The fact that patient 2 had an abnormal echocardiogram suggests that this may be an explanation for the increased cardiac markers in her case. Second, B units of CK can be abnormally produced by regenerating skeletal muscle when these patients are treated with steroids. ${ }^{910}$ Troponin $\mathrm{T}$ can also be produced from skeletal and myocardial muscle during the active phase of inflammatory muscle disease, and may reflect subclinical myocardial damage. ${ }^{211}$ Another possibility is that there could be problems with the non-specificity of the troponin $\mathrm{T}$ assay, with crossreactivity between the cardiac and skeletal isoforms of troponin T. However, the manufacturer's information regarding this third generation troponin $\mathrm{T}$ kit states that such crossreactivity is negligible. The final possible explanation is the potential re-expression of the cardiac troponin isoform in skeletal muscle; however, this is controversial, and it is important to note that there are particular issues in translating immunohistochemical studies from tissue sections of skeletal muscle to serum assays. ${ }^{12}$

\section{"Troponin concentrations must be interpreted in their clinical context"}

These two cases underline the need to recognise the shortfalls of troponin testing and the importance of not using troponin assays in isolation outside of the clinical decision making process. In retrospect, patient 1 was inappropriately admitted to hospital and overinvestigated. Inflammatory muscle disease (polymyositis and dermatomyositis) is an important cause of increased cardiac markers (CK-MB and troponins $)^{2-4}$; ; these are raised in up to $75 \%$ of patients. ${ }^{11}$ Our laboratory protocol of measuring troponin $\mathrm{T}$ concentrations automatically when CK-MB is raised contributed to the problem; in the future, this will not be done unless specifically requested by the patient's attending clinician.

In conclusion, we have described two case reports of patients suffering from inflammatory muscle disease with raised concentrations of cardiac markers (CK-MB and troponin T) in the absence of acute myocardial damage. The increased CK-MB and troponin $\mathrm{T}$ concentrations were caused by inflammatory muscular disease. However, we cannot be sure of the exact source of the raised cardiac markers; they could indicate myocardial involvement by the inflammatory process or a non-cardiac (probable skeletal muscle) source. The experience gained from the diagnosis and management of the first patient was invaluable when the second patient presented.

These cases outline the importance of considering inflammatory muscle disease as a cause of increased troponin $\mathrm{T}$, in the absence of clinical evidence of an acute coronary ischaemic event. Troponin concentrations must be interpreted in their clinical context. They also highlight the role of the clinical biochemistry laboratory in the education of clinical colleagues regarding the appropriate use of troponin assays and the interpretation of results.

\section{Authors' affiliations}

J S Hamilton, Department of Clinical Biochemistry, Royal Victoria Hospital, Belfast BT12 6BA, Northern Ireland, UK

P C Sharpe, Department of Clinical Biochemistry, Craigavon Area Hospital Group Trust, 68 Lurgan Road, Portadown BT63 5QQ, UK

Correspondence to: Dr P C Sharpe, Clinical Biochemistry, Craigavon Area Hospital Group Trust, 68 Lurgan Road, Portadown BT63 5QQ, UK; pcsharpe@cahgt.n-i.nhs.uk

Accepted for publication 14 March 2005

\section{REFERENCES}

1 Ammann P, Pfisterer $M$, Fehr T, et al. Raised cardiac troponins. BMJ 2004;328:1028-9.

2 White GH, Tideman PA. Increased troponin T in a patient with dermatomyositis. Clin Chem 2001;47:1130-1.

3 Erlacher $\mathbf{P}$, Lercher A, Falkensammer J, et al. Cardiac troponin and beta-type myosin heavy chain concentrations in patients with polymyositis or dermatomysitis. Clin Chim Acta 2001;306:27-33.

4 Kobayashi S, Tanaka M, Tamura N, et al. Serum cardiac troponin T in polymyositis/dermatomyositis. Lancet 1992;340:726.

5 Collinson PO, Boa FG, Gaze DC. Measurement of cardiac troponins. Ann Clin Biochem 2001;38:423-49

6 Gerhardt W, Liungdahl L. Troponin T: a sensitive and specific diagnostic and prognostic marker of myocardial damage. Clin Chim Acta 1998;272:47-57.

7 Hammerer-Lercher A, Erlacher P, Bittner R, et al. Clinical and experimental results on cardiac troponin expression in Duchenne muscular dystrophy. Clin Chem 2001;47:451-8.

8 Collinson PO, Stubbs PJ. Are troponins confusing? Heart 2003;89:1285-7.

9 Kiely PD, Bruckner FE, Nisbet JA, et al. Serum skeletal troponin I in inflammatory muscle disease: relation to creatine kinase, CKMB and cardiac troponin I. Ann Rheum Dis 2000;59:750.

10 Bodor GS, Survant L, Voss EM, et al. Cardiac troponin T composition in normal and regenerating human skeletal muscle. Clin Chem 1997:43:476-84.

11 Prellwitz W, Hafner G, Rupprecht HJ, et al. Diagnostic and differential diagnostic value of troponins. Med Klin 1996;91:336-42.

12 Fredericks S, Bainbridge K, Murray JF, et al. Measurement of cardiac troponin I in striated muscle using three experimental methods. Ann Clin Biochem 2003;40:244-8. 